\title{
Design of Modulation and Packet Detection in Body Area Networks
}

\author{
Guoquan Li, Ke Tang, Jinzhao Lin, Yu Pang, Zhangyong Li, Qianneng Zhou, Lu Deng \\ Chongqing University of Posts and Telecommunications, Chongqing, China \\ Email: ligq@cqupt.edu.cn
}

\begin{abstract}
In burst communication systems like Body Area Networks (BAN), burst packet should be detected prior to the demodulation of the signal at the receiver. Meanwhile, different modulation and demodulation algorithms may affect the BER performance of the entire system seriously. To satisfy the conditions that BAN nodes or devices have to be small in size and very low in power consumption, algorithm design of modulation and packet detection are very important. Referring to IEEE 802.15.6 BAN standard, theoretical analysis of the modulation and demodulation for $\pi / 2$-DBPSK and $\pi / 4$-DQPSK is carried out first. The two modulations are realized by using two different two-step looking up table respectively and then united to be one by combining the two phase increment tables. By use of the special structure of $\pi / 2$-DBPSK modulated preamble, packet detection algorithm with low complexity is also carefully designed. Simulation results of BER performance are presented at last. In AWGN channel, both modulations achieve good performance, and performance degradation of $\pi / 4$-DQPSK happens in multipath channel which may not satisfy the requirements. Adaptive modulation is considered to solve this problem by choosing different modulation scheme according to channel quality.
\end{abstract}

Index Terms-Body Area Network; Modulation; Demodulation; Packet Detection; Bit Error Rate

\section{INTRODUCTION}

B ODY Area Network (BAN) is an emerging hot research area in short-range wireless communication. It is a communication network which is composed of various network nodes distributed in, on or around human body [1]-[3], including personal terminal, sensors and networking devices. Currently, BAN is mainly used in patient care and physiological parameters measurement [4]-[6]. Through BAN, the measured parameters can be transferred to portable personal electronic devices such as PDA, mobile phones and even remote information center in the hospital for medical care or treatment. Thus it has a very broad application prospect.

Digital modulation and demodulation techniques have significant influence on the system bit error rate (BER)

This work was supported in part by the Special Project of Internet of Things from Ministry of Industry and Information Technology, 2013 University Innovation Team Construction Plan Funding Project of Chongqing, the National Science Foundation of China (Grant No. $61102075,61301124,61301125)$, the Chongqing Development Plan of Innovative Young Talents(Grant No. cstc2013kjrc-qnrc0126), the Key Program of Natural Science Foundation of Chongqing (Grant No. CSTC2013JJB40004), the Natural Science Foundation of Chongqing (Grant No. CSTCJJA40011), and the Board of Chongqing Education of Science and Technology Research Project (Grant No. KJ120507 and KJ120503). performance. As two commonly used modulations, BPSK and QPSK are easy to be realized, and also have excellent anti-interference ability and strong anti-fading performance [7]. Therefore, they are widely used in shortrange wireless communication systems [8]-[10]. Since BAN nodes has to be small, intelligent, low in power consumption and so on, high-complexity techniques should be avoided in the realization. Since BPSK and QPSK has the problem of phase ambiguity, $\pi / 2$-shift differentially encoded BPSK ( $\pi / 2$-DBPSK) and $\pi / 4$-shift differentially encoded QPSK ( $\pi / 4$-DQPSK) are adopted in BAN standard, IEEE 802.15.6 [11]. Both of them can be noncoherently demodulated and thus have low complexity. Moreover, The BAN channel in or around human body is complicated, the modeling of channel characteristics is still under research [12], [13].

For a burst communication system, like BAN, packet should be detected before the signal is modulated and decoded at the receiver. To achieve rapid packet detection, a preamble sequence located in the beginning of a packet, is generally adopted [14]-[18]. Many detectors have been put forward including the energy detector [18] and the cross-correlation detector [17] for the packet detection in AWGN channel. [14] investigates burst packet detection in multipath environment and proposes a cascaded matched filter-autocorrelation (MF-AC) detector to strengthen the detection performance in multi-path channel. However, the preamble of BAN data frame is modulated with $\pi / 2$-DBPSK and has special structure. Therefore, more information about the preamble can be exploited to reduce the complexity, which is particularly important for low-power-consumption BAN devices.

Considering that little research on the performance and demodulation algorithm has been done in BAN systems, BER of $\pi / 2$-DBPSK and $\pi / 4$-DQPSK is theoretically analyzed and simulated based on Monte Carlo statistical method in the paper. A packet detection method special for BAN systems is also proposed and analyzed.

\section{Algorithm PRINCIPLE}

At the transmitter, the phase modulated signal is expressed as

$$
\begin{aligned}
s(t) & =A \cos \left(\omega_{c} t+\theta\right) \\
& =A\left[\cos \left(\omega_{c} t\right) \cos \theta-\sin \left(\omega_{c} t\right) \sin \theta\right],
\end{aligned}
$$

where $\omega_{c}$ is the carrier frequency, $\theta$ is the transient phase value of phase modulated signal. 
In differential phase modulation mode, the absolute phase $\theta$ of the current symbol changes over time and associates with the previous absolute phase. The absolute phase of the symbol at time $k$ is expressed as

$$
\theta_{k}=\theta_{k-1}+\Delta \theta_{k},
$$

so the following equations can be obtained from (2),

$$
\begin{aligned}
& \cos \left(\theta_{k}\right)=\cos \left(\theta_{k-1}\right) \cos (\Delta \theta)-\sin \left(\theta_{k-1}\right) \sin (\Delta \theta) \\
& \sin \left(\theta_{k}\right)=\sin \left(\theta_{k-1}\right) \cos (\Delta \theta)+\cos \left(\theta_{k-1}\right) \sin (\Delta \theta)
\end{aligned}
$$

According to (3) and (4), current cosine value can be represented by the cosine value of previous symbol phase and phase increment. The signal after I/Q separation is expressed as

$$
I_{k}=\cos \theta_{k}, Q_{k}=\sin \theta_{k} .
$$

Then (3) and (4) are rewritten as

$$
\begin{aligned}
& I_{k}=I_{k-1} \cos (\Delta \theta)-Q_{k-1} \sin (\Delta \theta), \\
& Q_{k}=Q_{k-1} \cos (\Delta \theta)+I_{k-1} \sin (\Delta \theta) .
\end{aligned}
$$

At the demodulator, it is assumed that the local oscillator and transmitter carrier have the same frequency and a fixed phase difference $\psi$ so that

$$
W_{k}=\cos \left(\theta_{k}-\psi\right), Z_{k}=\sin \left(\theta_{k}-\psi\right) .
$$

Using $\pi / 2$-DBPSK modulation, let

$$
e_{k}=W_{k-1} Z_{k}-W_{k} Z_{k-1}=\sin \Delta \theta .
$$

If $e_{k}>0$, then 0 is decided, otherwise it is 1 . Using $\pi / 4$-DQPSK modulation [19], let

$$
\begin{aligned}
& f_{k}=W_{k} W_{k-1}-Z_{k} Z_{k-1}=\cos \Delta \theta, \\
& g_{k}=W_{k-1} Z_{k}-W_{k} Z_{k-1}=\sin \Delta \theta
\end{aligned}
$$

If $f_{k}>0, g_{k}>0$, then 00 sequence is decided. Similarly, we know $01,10,11$ are transmitted when $f_{k}<0$ and $g_{k}>0, f_{k}>0$ and $g_{k}<0, f_{k}<0$ and $g_{k}<0$ respectively.

\section{APPLICATIONS IN BAN}

\section{A. Modulation}

In this paper, we realized the modulation in BAN by looking up the table of absolute phase $\theta$ and phase increment $\Delta \theta$. As in IEEE 802.15.6 standard, the initial phase is initialized to $\pi / 2$. The modulation parameters for 2400-2483.5 frequency band are shown in TABLE.I. $\pi / 2$ DBPSK and $\pi / 4$-DQPSK are selected respectively [5] according to the different data rates and packet components. For $\pi / 2$-DBPSK, $\Delta \theta$ belongs to $\{\pi / 2,3 \pi / 2\}$. For $\pi / 4$ DQPSK, $\Delta \theta$ takes value from $\{\pi / 4,3 \pi / 4,5 \pi / 4,7 \pi / 4\}$. Comparing to the traditional BPSK and QPSK, they both have phase change in every symbol time which improves the system performance. The relationship between phase increment $\Delta \theta$ and information bit $b(n)$ is shown in TABLE.II and TABLE.III.
TABLE II.

$\pi / 2$-DBPSK PHASE INCREMENT

\begin{tabular}{c|c}
\hline \hline$b(n)$ & $\Delta \theta$ \\
\hline 0 & $\pi / 2$ \\
1 & $3 \pi / 2$ \\
\hline
\end{tabular}

TABLE III.

$\pi / 4$-DQPSK PHASE INCREMENT

\begin{tabular}{c|c|c}
\hline \hline$b(2 n)$ & $b(2 n+1)$ & $\Delta \theta$ \\
\hline 0 & 0 & $\pi / 4$ \\
0 & 1 & $3 \pi / 4$ \\
1 & 0 & $7 \pi / 4$ \\
1 & 1 & $5 \pi / 4$ \\
\hline
\end{tabular}

Let $N$ be the absolute phase number of current symbol, and $N^{\prime}$ be the number of previous one. The absolute phase $\theta$ of two modulations are shown in TABLE.IV and TABLE.V. According to (6) and (7), current $\theta$ can be decided by the previous one and phase increment. For $\pi / 2$-DBPSK, if

$$
\Delta \theta=k \pi / 2(k=1,3),
$$

then

$$
N=\bmod \left(N^{\prime}+k, 4\right) .
$$

For $\pi / 4-\mathrm{DQPSK}$, if

$$
\Delta \theta=k \pi / 4(k=1,3,5,7)
$$

then

$$
N=\bmod \left(N^{\prime}+k, 8\right) .
$$

Finally, the modulation process is completed by looking up the two tables.

Seen from TABLE.I, $\pi / 2$-DBPSK and $\pi / 4$-DQPSK are both used in BAN according to the different data rates and packet components. Considering that two modulations are very similar, a united modulation can be constructed with a selection port indicating the specific modulation mode. Combing TABLE.II and TABLE.III, a new table is obtained as TABLE.VI. For $\pi / 2$-DBPSK, $b(2 n)$ and $b(2 n+1)$ take the same value of the only input information bit before looking up TABLE.VI.

By using TABLE.VI, $\pi / 2$-DBPSK seems to be transformed to $\pi / 4$-DQPSK, so the absolute phase obtained from the phase increment $\Delta \theta$ must take value from $\{0, \pi / 4, \pi / 2,3 \pi / 4, \pi, 5 \pi / 4,3 \pi / 2,7 \pi / 4\}$ and TABLE.V can still be used to realize the modulation.

TABLE IV.

$\pi / 2$-BPSK ABSOLUTE PHASE

\begin{tabular}{c|c|c|c}
\hline \hline$N$ & $\theta$ & $I_{k}$ & $Q_{k}$ \\
\hline 0 & 0 & 1 & 0 \\
1 & $\pi / 2$ & 0 & 1 \\
2 & $\pi$ & -1 & 0 \\
3 & $3 \pi / 2$ & 0 & -1 \\
\hline
\end{tabular}


TABLE I.

MODULATION PARAMETERS

\begin{tabular}{c|c|c|c|c|c|c|c}
\hline \hline $\begin{array}{c}\text { Packet } \\
\text { component }\end{array}$ & $\begin{array}{c}\text { Modulation } \\
\text { mode }\end{array}$ & $\begin{array}{c}\text { Symbol rate } \\
(k b p s)\end{array}$ & $\begin{array}{c}\text { Code rate } \\
(k / n)\end{array}$ & $\begin{array}{c}\text { Spreading } \\
\text { factor }\end{array}$ & $\begin{array}{c}\text { Pulse } \\
\text { shape }\end{array}$ & $\begin{array}{c}\text { Information } \\
\text { data rate }(k b p s)\end{array}$ & Support \\
\hline PLCP & $\pi / 2$-DBPSK & 91.9 & $19 / 32$ & 4 & SRRC & 91.9 & Mandatory \\
PSDU & $\pi / 2$-DBPSK & 121.4 & $51 / 63$ & 4 & SRRC & 121.4 & Mandatory \\
PSDU & $\pi / 2$-DBPSK & 242.9 & $51 / 63$ & 2 & SRRC & 242.9 & Mandatory \\
PSDU & $\pi / 2$-DBPSK & 485.7 & $51 / 63$ & 1 & SRRC & 485.7 & Mandatory \\
PSDU & $\pi / 4$-DQPSK & 971.1 & $51 / 63$ & 1 & SRRC & 971.4 & Mandatory \\
\hline
\end{tabular}

TABLE V. $\pi / 4$-QPSK ABSOLUTE PHASE

\begin{tabular}{c|c|c|c}
\hline \hline$N$ & $\theta$ & $I_{k}$ & $Q_{k}$ \\
\hline 0 & 0 & 1 & 0 \\
1 & $\pi / 4$ & 0.707 & 0.707 \\
2 & $\pi / 2$ & 0 & 1 \\
3 & $3 \pi / 4$ & -0.707 & 0.707 \\
4 & $\pi$ & -1 & 0 \\
5 & $5 \pi / 4$ & -0.707 & -0.707 \\
6 & $3 \pi / 2$ & 0 & -1 \\
7 & $7 \pi / 4$ & 0.707 & -0.707 \\
\hline
\end{tabular}

TABLE VI

PHASE INCREMENT FOR $\pi / 2$-DBPSK AND $\pi / 4$-DQPSK UNITED MODULATION

\begin{tabular}{c|c|c|c}
\hline \hline selection & $b(2 n)$ & $b(2 n+1)$ & $\Delta \theta$ \\
\hline 0 & 0 & 1 & $\times$ \\
0 & 1 & 0 & $\times$ \\
0 & 0 & 0 & $2 \pi / 4$ \\
0 & 1 & 1 & $6 \pi / 4$ \\
1 & 0 & 0 & $\pi / 4$ \\
1 & 0 & 1 & $3 \pi / 4$ \\
1 & 1 & 0 & $7 \pi / 4$ \\
1 & 1 & 1 & $5 \pi / 4$ \\
\hline
\end{tabular}

\section{B. Packet Detection}

In BAN, a preamble is added prior to the PLCP header in order to aid the receiver in packet detection, timing synchronization and carrier-offset recovery. Two unique preambles are defined in order to mitigate false alarms due to networks operating on adjacent channels [11]. Each preamble is constructed by concatenating a length63 m-sequence with a 010101010101101101101101101 extension sequence. The former sequence is used to implement packet detection, coarse-timing synchronization and carrier-offset recovery, while the latter sequence can be used to implement fine-timing synchronization. Meanwhile, the preamble is transmitted at the same symbol rate and encoded using the same modulation parameters as defined for the PLCP header in TABLE.I, which means $\pi / 2$-DBPSK is adopted for the preamble.

Assume that the oversampling rate is $M$ and the received signal is

$$
r_{k}=W_{k}+j Z_{k}
$$

Traditionally, the preamble should be recovered from the received modulated signal $r_{k}$ and differential phase is removed at first. However, the difference operation at the receiver will lead to noise enhancement. Therefore, in this paper, local reference sequence is modulated using $\pi / 2$-DBPSK instead of the difference of received signal. Then the correlation operation is done between $r_{k}$ and modulated local reference sequence, defined as $p_{k}$. Let

$$
p_{k}=A_{k}+j B_{k}
$$

From TABLE.IV, we know that one of the in-phase branch $I_{k}$ and quadrature branch $Q_{k}$ of the $\pi / 2$-DBPSK modulated signal must be 0 at any time $k$. Taking preamble sequence $\sharp 1$ as an example, the following equations can be obtained

$$
A_{2 n}=B_{2 n-1}=0,(n=1,2, \cdots, 31) .
$$

Calculate the complex correlation of $r_{k}$ and $p_{k}$ as shown in Fig.1, and then the correlation value will be

$$
R=\sum_{l=1}^{63} r_{l} \times p_{l}^{*},
$$

where $(\cdot)^{*}$ represents the conjugate of a complex number.

Combine (12), (13) with (15),

$$
\begin{aligned}
R & =\sum_{l=1}^{63}\left(W_{l}+j Z_{l}\right)\left(A_{l}-j B_{l}\right) \\
& =\sum_{l=1}^{63}\left[\left(W_{l} A_{l}+Z_{l} B_{l}\right)+j\left(Z_{l} A_{l}-W_{l} B_{l}\right)\right]
\end{aligned}
$$

Let $I^{\prime}$ and $Q^{\prime}$ denote the real and imaginary part of $R$ respectively. By using (14),

$$
\begin{aligned}
I^{\prime} & =\sum_{n=1}^{32}\left(W_{2 n-1} A_{2 n-1}+Z_{2 n-1} B_{2 n-1}\right) \\
& +\sum_{n=1}^{31}\left(W_{2 n} A_{2 n}+Z_{2 n} B_{2 n}\right) \\
& =\sum_{n=1}^{32}\left(W_{2 n-1} A_{2 n-1}\right)+\sum_{n=1}^{31}\left(Z_{2 n} B_{2 n}\right)
\end{aligned}
$$




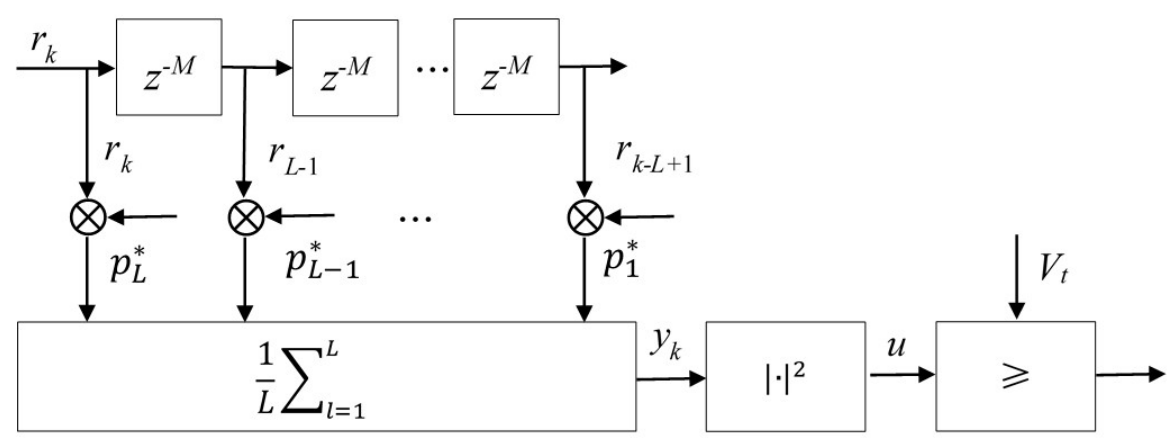

Figure 1. Packet detection algorithm schematic.

$$
\begin{aligned}
Q^{\prime} & =\sum_{n=1}^{32}\left(Z_{2 n-1} A_{2 n-1}-W_{2 n-1} B_{2 n-1}\right) \\
& +\sum_{n=1}^{31}\left(Z_{2 n} A_{2 n}-W_{2 n} B_{2 n}\right) \\
& =\sum_{n=1}^{32}\left(Z_{2 n-1} A_{2 n-1}\right)-\sum_{n=1}^{31}\left(W_{2 n} B_{2 n}\right)
\end{aligned}
$$

Since both $A_{k}$ and $B_{k}$ takes value 1 or -1 , only add or subtract will be enough to calculate (17) and (18) and hence the complexity is very low. Considering that $R$ is a complex number, the packet can be detected by comparing the energy $u$ as follows with a threshold.

$$
\begin{aligned}
u & =|R / 63|^{2} \\
& =\frac{1}{3969}\left[\left(I^{\prime}\right)^{2}+\left(Q^{\prime}\right)^{2}\right] .
\end{aligned}
$$

\section{Simulation ANALYSis}

In this section, BER of $\pi / 2$-DBPSK and $\pi / 4$-DQPSK in AWGN channel and multipath Rayleigh fading channel are simulated. Path number for multipath channel is 4 . The system sampling rate $T_{s}=1 / 2.4 \mu \mathrm{s}$. Mean and variance of Gaussian random variable are 0 and $\delta_{k}^{2}$ respectively.

At the transmitter, data frame is generated by concatenating PLCP preamble and PLCP header with random binary sequence and then processed as shown in Fig.2 to realize the modulation. A mod operation is used to avoid the absolute phase exceeding $2 \pi$. Finally, I branch and $\mathrm{Q}$ branch are obtained by looking up the tables. After receiving the noised I and $\mathrm{Q}$ data: $W_{k}$ and $Z_{k}$, packet detection and carrier recovery is done first, and then demodulation and decision are applied as (8) to (11) to acquire the random binary sequence. BER Monte Carlo statistics in AWGN channel and multipath channel are shown in Fig. 3 and Fig.4 respectively.

Seen from Fig.3, when $E_{b} / N_{0}=10 d B$, both modulations have BER lower than $10^{-6}$, which satisfies the general engineering requirements for data transmission. Although $\pi / 2$-DBPSK obtains $1 d B$ gain over $\pi / 4$-DQPSK, $\pi / 4$-DQPSK has approximately double bandwidth efficiency [20]. As in Fig.4, when $\pi / 2$-DBPSK is selected, BER performance in multipath channel deteriorates $2 d B$ over that in AWGN channel. For $\pi / 4$-DQPSK, it is $2.5 \mathrm{~dB}$, and thus may not meet the demands.
In practice, the presence of multipath fading channel is almost inevitable because of complex human body channel [21]. Therefore, adaptive modulation can be considered according to channel quality. $\pi / 4$-DQPSK switches to $\pi / 2$-DBPSK automatically when channel deteriorates to obtain good communication quality.

\section{CONCLUSIONS}

According to IEEE 802.15.6 BAN standard, theoretical research and simulation analysis of $\pi / 2$-DBPSK and $\pi / 4$ DQPSK are carried out based on Monte Carlo statistical method. Packet detection based on the special structure of $\pi / 2$-DBPSK modulated preamble is also designed and proved to be low complexity. Simulations show that both modulations meet the requirements in AWGN channel and $\pi / 4$-DQPSK obtains approximately double bandwidth efficiency. However, in multipath channel, BER performance of $\pi / 4$-DQPSK is worse than $\pi / 2$-DBPSK, which may not reach the performance requirements. Considering this, an adaptive modulation strategy can be adopted to choose different modulation with channel fluctuation to keep good communication quality. Based on the special structure of $\pi / 2$-DBPSK modulated preamble in IEEE 802.15.6 BAN, a packet detection method with low calculation complexity is also proposed and analyzed.

\section{ACKNOWLEDGMENT}

The authors are grateful to the anonymous referees for their valuable comments and suggestions to improve the presentation of this paper.

\section{REFERENCES}

[1] Karaoguz J, "High rate wireless personal area networks," IEEE Comm. Magazine, vol.39, pp. 96-102, Dec. 2001.

[2] "IEEE Standard for Telecommunications and Information Exchange Between Systems - LAN/MAN Specific Requirements - Part 15.3: Wireless Medium Access Control (MAC) and Physical Layer (PHY) Specs for High Rate Wireless Personal Area Networks," 2007.

[3] I. F. Akyildiz , W. Su , Y. Sankarasubramaniam and E. Cayirci, "Wireless sensor networks: A survey," Computer Networks, vol. 38, pp. 393-422 Mar. 2002.

[4] M. Patel and J. Wang, "Applications, challenges, and prospective in emerging body area networking technologies," IEEE Wireless Communication Magzine, vol. 17, pp.80-88, Jan. 2010. 


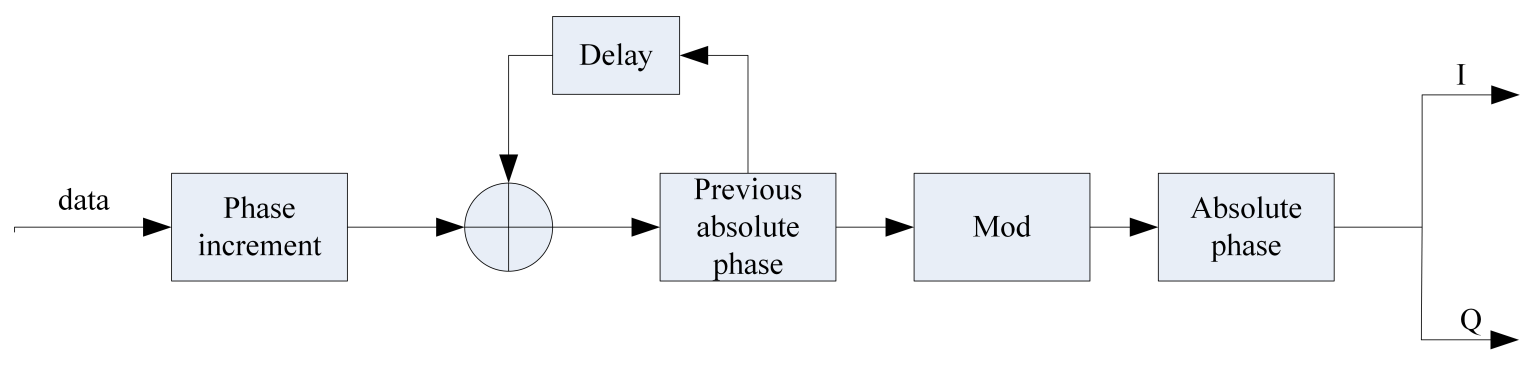

Figure 2. Table looking up process.

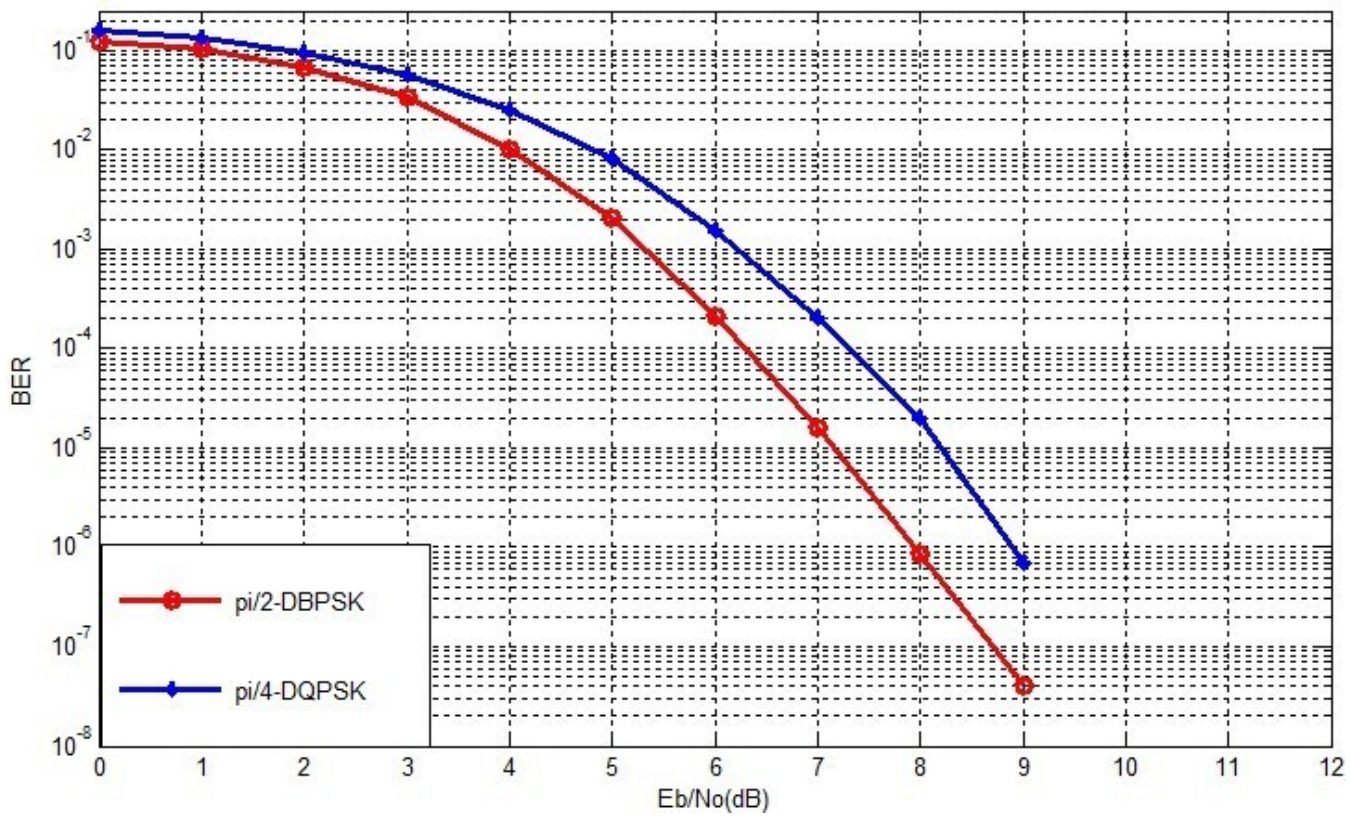

Figure 3. BER curve in AWGN channel.

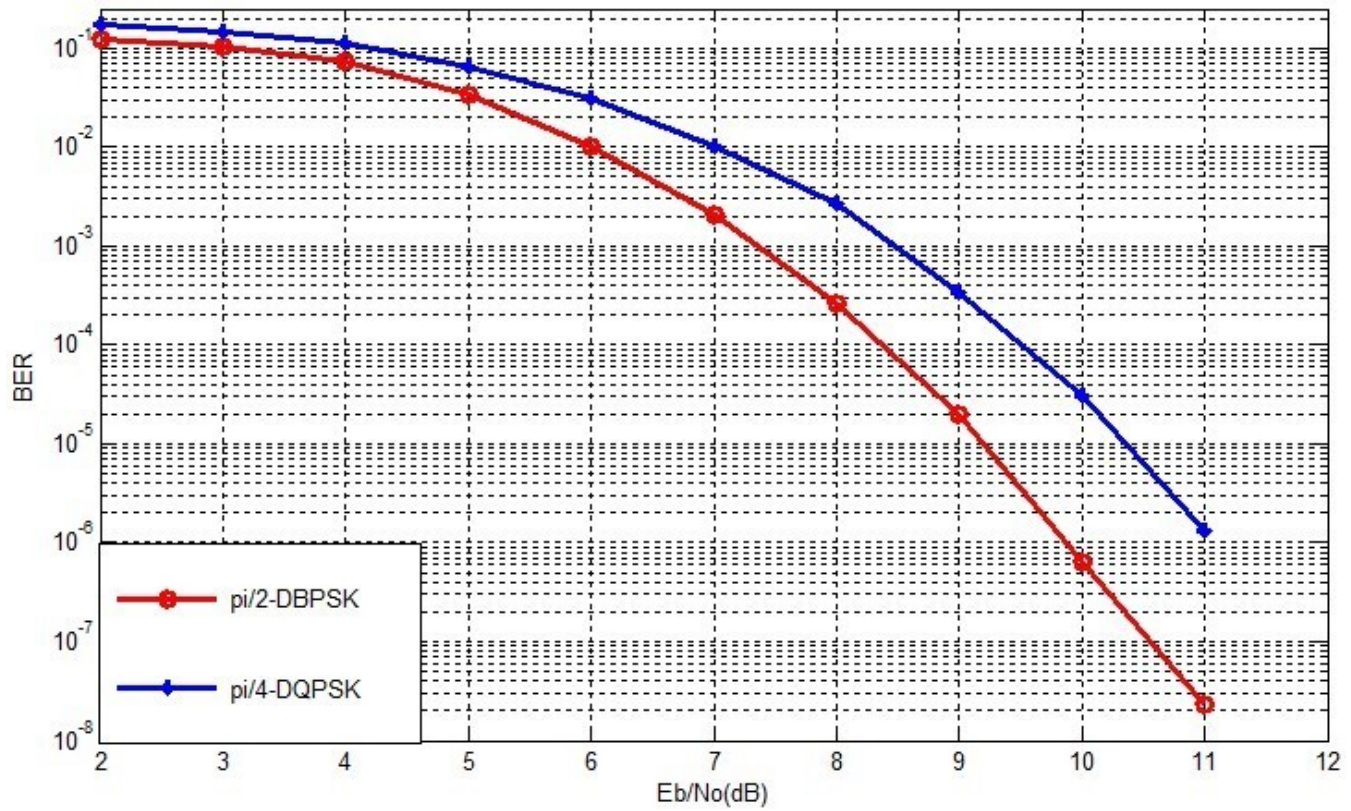

Figure 4. BER curve in multipath channel. 
[5] M. Chen , S. Gonzalez, A. Vasilakos , H. Cao and V. C. M. Leung "Body area networks: A survey," Mobile Networks and Applications, vol. 16, pp.171-193, Apr. 2010.

[6] Won-Jae Yi, Sufeng Niu, Gonnot, T., Saniie, J., "System architecture of intelligent personal communication node for body sensor network," 2013 IEEE International Instrumentation and Measurement Technology Conference, Minneapolis, pp. 1717-1720, May 2013.

[7] Changxing Fan and Lina Cao, Communication Theory, $6^{t h}$ edition, Beijing national defense industry publishing house, 2008, pp. 180-237. In Chinese

[8] Y. S. Poberezhskly, "Novel modulation techniques and circuits for transceivers in Body sensor netwirks," IEEE Journal on Emerging and Selected Topics in Circuits and Systems, Vol.2, pp.96-108, Mar. 2012.

[9] Natarajan K., Allstot, D.J., Walling, J.S., "Transmitters for body sensor networks: A comparative study," 2011 IEEE Biomedical Circuits and Systems Conference (BioCAS), San Diego, pp. 185-188, Nov. 2011.

[10] Vasanthi M.S., Kumar D., Rama Rao T., ”Analysis of AQDBPSK modulation for WSN transceivers in indoor environments," 2013 International Conference on Information Communication and Embedded Systems, Chennai, pp. 904907, Feb. 2013.

[11] IEEE Standard for Local and metropolitan area networks - Part 15.6: Wireless Body Area Networks, 2012.

[12] Bo Xu and Pengfei He, "Wireless Body Area Network Channel Characteristics," Telecommunications Science, vol.27, pp. 81-82, Mar. 2011. In Chinese

[13] G. Noetscher, S. Makarov, and N. Clow, "Modeling accuracy and features of body-area networks with out-of-body antennas at $402 \mathrm{MHz}, "$ IEEE Antennas and Propagation Magazine, vol. 53, pp. 118-143, Aug. 2011.

[14] Z. Xiao, D. Jin, and N. Ge, "Matched FilterAutocorrelation (MF-AC) for Packet Detection in Multipath Channels," IEEE Communications Letters, vol. 17, pp. 1608-1611, Aug. 2013.

[15] K. M. Ok, and C. G. Kang, Generalized Window-Based PN Acquisition Scheme in CDMA Spread Spectrum Systems, IEEE Transactions on Wireless Communications, vol. 7, pp. 2851-2855, Aug. 2008.

[16] I.-T. Lu, and K.-J. Tsai, "A novel start-of-packet detection and synchronization scheme." proceedings of the 201134 th IEEE Sarnoff Symposium, Princeton, pp. 1-5, May 2011.

[17] Z. Xiao, D. Jin, and L. Zeng, "Normalized-energy-detector serial search acquisition scheme for packet communication systems with non-ideal AGC," Wireless Personal Communication, vol. 65, pp. 895C907, Apr. 2012.

[18] K. Bai, N. He, and C. Tepedelenlioglu, "Acquisition performance comparison of energy detector and singlepulse correlator for UWB systems," Proceedings of 41 st Annual Conference on Information Sciences and Systems, Baltimore, pp. 141C146, Mar. 2007.

[19] Song Huang, " $\pi / 4$-DQPSK Baseband differential demodulation techniques and data transmission implementation," University of Electronic Science and Technology, pp. 2240, 2006.In Chinese

[20] Yumei Ding and Xiquan Gao, Digital Signal Processing, Second edition, Xi'an university of electronic science publishing house, pp. 195-210, 2001. In Chinese

[21] Chunmei Yan and Xiaorong lv, "Mobile Medical Services Technology Progress and prospects," Transducer and Microsystem, pp. 1-6, Feb. 2013. In Chinese

Guoquan Li received his Ph.D. degree in College of Communication Engineering from Chongqing University (CQU), Chongqing, China in 2012. He is currently a lecturer with Chongqing University of Posts and Telecommunications, Chongqing (CQUPT), China. His research interests include digital baseband signal processing, multi-user MIMO system, coding theory and technology.

Ke Tang is currently a college student and working towards his B.S. degree at Chongqing University of Posts and Telecommunications, China. His current research interest includes shortrange wireless communication systems and baseband signal processing.

Jinzhao Lin received Ph.D degree in 2001 from Chongqing University. Since 2003 he has been a professor with Chongqing University of Posts and Telecommunications (CQUPT). His current interests include wireless communications and ASIC design.

Yu Pang received Ph.D degree in 2010 from McGill University. Since 2012 he has been a professor with Chongqing University of Posts and Telecommunications (CQUPT). His current interests include wireless communications and ASIC design.

Zhangyong Li received Ph.D degree in 2004 from Chongqing Medical University. Since 2008 he has been a professor with Chongqing University of Posts and Telecommunications (CQUPT). His current interests include digital signal processing and biology engineering.

Qianneng Zhou received the B.S. degree, the M.Sc degree, and $\mathrm{Ph} . \mathrm{D}$ degree, all in microelectronic, from Harbin Institute of Technology (HIT), Harbin, China, in 1998, 2002 and 2009, respectively. Since 2009, he has been an associate professor with Chongqing University of Posts and Telecommunications (CQUPT). His current interests include dc-dc converters, amplifiers, LDO regulator and LED drivers.

Lu Deng received the B.S. degree in wireless communication from Chongqing University of Posts and Telecommunications (CQUPT) in 2011. She is currently working toward the M.Sc degree at CQUPT. Her research interest is digital signal processing. 